\title{
Initial Performance Testing of Srl Gamma Spectroscopy Scintillators and Comparison to Other Improved-Resolution Detectors for Typical Health Physics Applications
}

\author{
F. L. Bronson CHP \\ Mirion Technologies - Canberra 800 Research Parkway Meriden CT USA
}

\begin{abstract}
Europium-doped Strontium lodide (Srl) scintillation gamma detectors have only recently become commercially available. Srl has very high light output ranging from $80,000-100,000 \mathrm{ph} / \mathrm{MeV}$ and energy resolution as good as $2.9 \%$ at $662 \mathrm{keV}$ and $14.8 \%$ at $32 \mathrm{keV}$. The high density of $4.6 \mathrm{~g} / \mathrm{cc}$ in combination with a high effective Atomic number and the absence of internal activity make Srl a very attractive sensor material for spectroscopic radiation monitoring devices. This new entrant into the field of commercially available gamma spectroscopy detectors offers improved energy resolution over Nal, without the elevated background of LaBr. While CZT and HPGe detectors have better resolution, the small available size of CZT and the cooling requirement of HPGe limit their usefulness in portable applications. Of these scintillators, Srl2 has the lowest detection limits when compared to other equalsize scintillation detectors, especially at low energies where $U$ and Pu are of interest.
\end{abstract}

\section{Introduction}

Strontium lodide was originally explored as a potential enhanced-resolution radiation detector by $\mathrm{R}$. Hofstadter in 1968. He expected promising results however the doping methods and crystal purity available at that time did not give satisfactory results. Strontium lodide was largely ignored until 2008 when scientists at Lawrence Livermore National Laboratory resurrected that effort and headed the research into enhanced-resolution scintillation detectors. That effort has now led to the availability of large-size good quality commercially available scintillators made from Europium-doped Strontium lodide (Srl2:Eu), hereafter abbreviated Srl.

Table 1 shows some of the characteristics of various gamma sensors. The features that make Srl attractive for gamma spectral measurements include:

- High light yield for better statistics

- Favorable wavelength for better matching to Photomultiplier Tubes

Table 1 Properties of various gamma detectors

\begin{tabular}{|c|c|c|c|c||}
\hline \multicolumn{5}{|c|}{ Detector Material Properties } \\
\hline \multirow{2}{*}{ Material } & $\begin{array}{c}\text { Density } \\
\text { g/cc }\end{array}$ & Z-Effective & $\begin{array}{c}\text { Light Yield } \\
\text { photons/MeV }\end{array}$ & $\begin{array}{c}\text { Peak Wavelength } \\
\mathrm{nM}\end{array}$ \\
\hline $\mathrm{Nal}(\mathrm{TI})$ & 3.67 & 32 & 38,000 & 415 \\
\hline $\mathrm{Srl}(\mathrm{Eu})$ & 4.59 & 48.2 & 80,000 & 435 \\
\hline $\mathrm{LaBr}(\mathrm{Ce})$ & 5.08 & 40.5 & 70,000 & 380 \\
\hline $\mathrm{CeBr}$ & 5.18 & 40.8 & 68,000 & 371 \\
\hline $\mathrm{CLYC}$ & 3.31 & 25.3 & 20,000 & 370 \\
\hline $\mathrm{CZT}$ & 5.78 & 42.4 & - & - \\
\hline $\mathrm{Ge}$ & 5.36 & 32 & - & - \\
\hline \hline
\end{tabular}

- High Effective Atomic Number for better stopping of gamma radiation

- Absence of internal radioactivity which limits the usefulness of scintillators containing La, due to the naturally radioactive La-138 it contains.

Another new scintillator coming out of the LLNL research efforts is Cs2LiYCl6 - thankfully commonly abbreviated as CLYC. It is also an enhanced resolution detector with also the ability to detect neutrons. 
A cursory evaluation of the detector material properties would also seem to favor $\mathrm{LaBr}$ and $\mathrm{CeBr}$ scintillators. Both have been commercially available for about $10 \mathrm{y}$. $\mathrm{LaBr}$ has demonstrated very good energy resolution - in the 3-3.5\% range, while $\mathrm{CeBr}$ has generally been in the $4-4.5 \%$ range. But the major drawback of $\mathrm{LaBr}$ is the internal radioactivity from the naturally occurring La-138. The graph in Figure 1 shows two similar size detectors in a shielded cave. The LaBr scintillator has a background nearly $10 x$ higher than the Srl. This is due to La-138. The higher energy peaks are due to residual radium impurities that are apparently quite difficult to remove. $\mathrm{CeBr}, \mathrm{Nal}$, and CLYC will all have background levels similar to that shown for Srl.

\section{Theoretical Calculations of Comparative Efficiency}

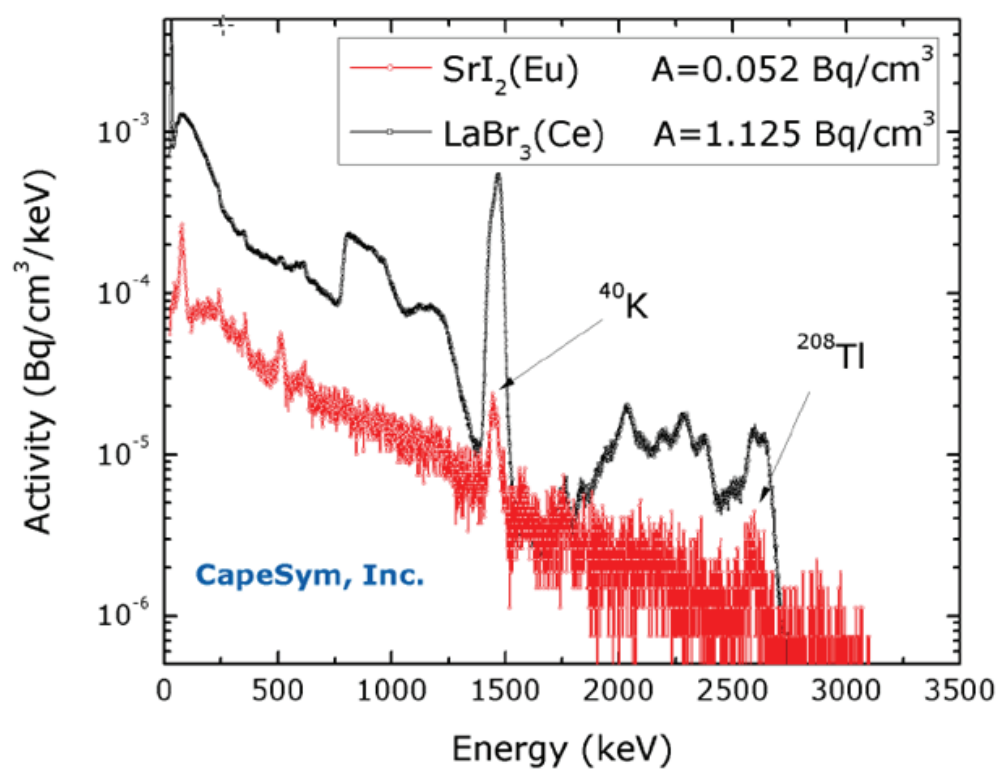

Figure 1 Shielded background for Srl and LaBr detectors of similar size

To obtain an early prediction of the potential performance of Srl, efficiency calculations were performed using the Mirion Technologies [Canberra] ISOCS software. Hypothetical perfect "radioactive sources" that were $51 \mathrm{~mm}$ diameter by $51 \mathrm{~mm}$ in length at $100 \mathrm{~mm}$ from a reference detector were modeled. Each "radioactive source" had the material composition and density of the tested scintillator. The modeled "radioactive sources" were: $\mathrm{Srl} 2, \mathrm{Nal}, \mathrm{LaBr}, \mathrm{CeBr}, \mathrm{CLYC}, \mathrm{CZT}$, and Ge, all common and available radiation detector materials. The ISOCS computed efficiency vs. energy is the product of the emission fraction from the "radioactive source", a geometrical factor, and the full energy peak absorption fraction of the detector. But since the detector is the same in all of these cases, ratio of the Nal emission fraction to the Srl emission fraction is equivalent to the ratio of the Srl efficiency to the Nal efficiency for that particular geometry. This is essentially the same concept as the Reciprocity Principle in radiation dosimetry where the source and the detector can be interchanged without changing the absorbed dose.

The following graphs in Figure 2 show the expected gamma peak efficiency of all of these "perfect" hypothetical detectors - as compared to the performance of Nal. The energy span is from $10 \mathrm{keV}$ to $7000 \mathrm{keV}$. All of these comparisons assume the same size detector. The left graph shows all of the data points for all of the materials. The right graph is an expanded view of the scintillators to better visualize the low energy performance.

Features that can be observed in these plots include:

- The much higher efficiency compared to Nal at 10-40 keV, due to the low Z-effective of Nal. CLYC is the exception as it has a low Z-effective.

- The higher efficiency of all of the scintillators [except CLYC] at all energies, due to the low density of Nal.

- Srl has the best efficiency in the energy range of 50-250 keV, due to the high Z-effective. The low Z-effective of CLYC and Ge is why their relative efficiency is low in the 50-300 keV range. 
- $\mathrm{LaBr}$ and $\mathrm{CeBr}$ have the best efficiency at $>250 \mathrm{keV}$ due to their high density.

- CZT shows the highest relative efficiency due to the highest density and high Z-effective and would make an excellent spectroscopy detector if it were ever available in large sizes.
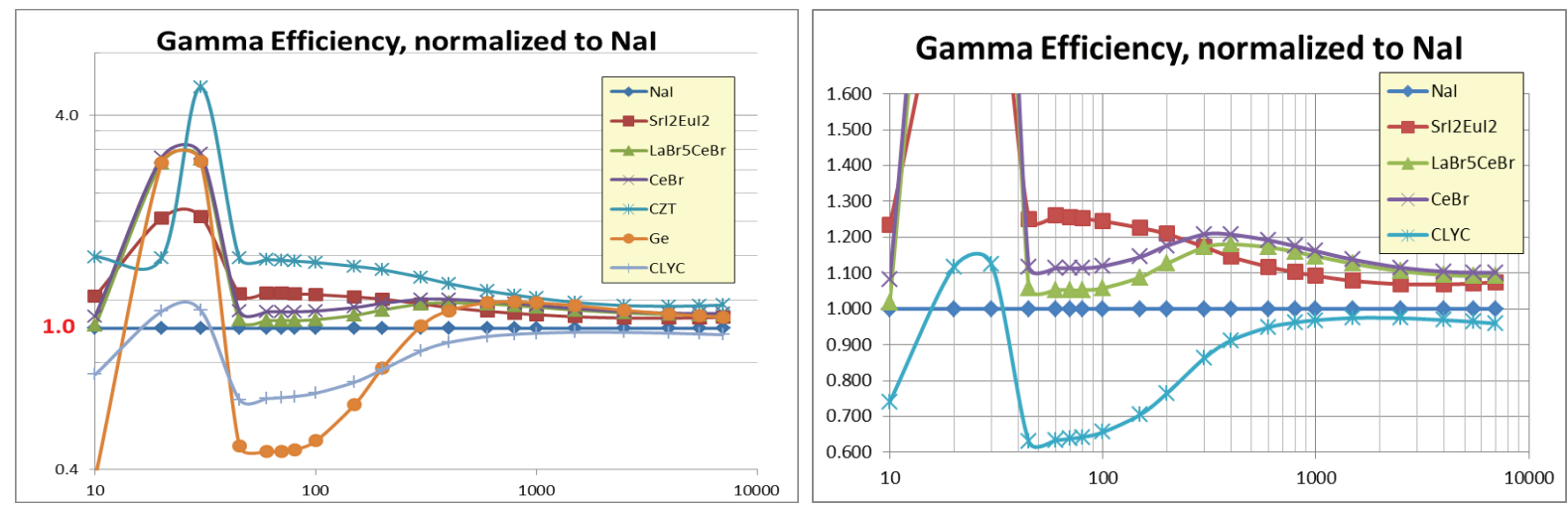

Figure 2 Gamma efficiency for equal size detectors, all normalized to Nal efficiency. The graph on the right is a vertically expanded scale of the graph on the left, and only for the enhanced resolution scintillators discussed in this document.

\section{Equipment Tested}

Two different sized Srl detectors were obtained, one is $38 \mathrm{~mm}$ diameter and $38 \mathrm{~mm}$ long, the other is $46 \mathrm{~mm}$ diameter and $26 \mathrm{~mm}$ long. Figure 3 shows these two detectors, along with a $3 \times 3$ Nal LED-stabilized detector and a 1cc CZT detector-MCA. The $38 \times 38 \mathrm{~mm}$ Srl detector is coupled to at 10-stage PMT which mostly explains why it is longer than the $46 \times 46 \mathrm{~mm}$ detector which is coupled to an 8-stage PMT.

The Mirion Osprey MCA is shown on the $38 \times 38 \mathrm{~mm}$ detector and was used in all of the scintillator tests.

Both of the Srl scintillators have essentially the same volume and therefore are approximately the same price. However the aspect ratio [ratio of diameter to thickness] of the $46 \times 26 \mathrm{~mm}$ detector gives it an efficiency advantage for most all geometries. This is the advantage long seen with the high aspect ratio Canberra BEGe detectors as compared the more traditional co-axial Ge detectors.

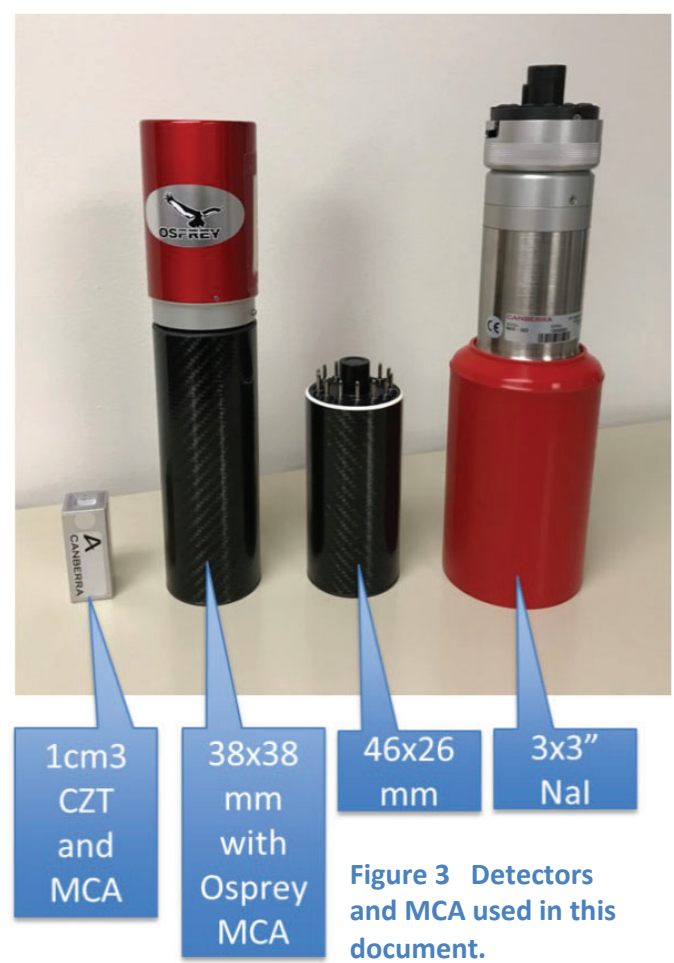


An ISOCS characterization was performed for both Srl detectors which allows us to quickly do efficiency calculations. Below in Figure 4 on the left is an energy-efficiency calibration comparison for a typical

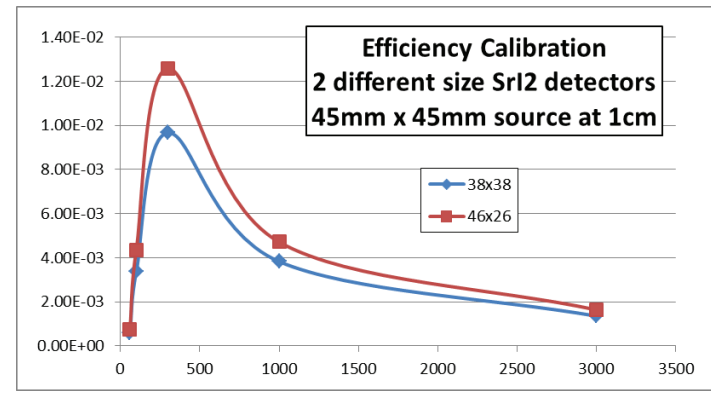

Figure 4 Left: Efficiency calibration for both detector sizes for a typical laboratory geometry. Right: Ratio of efficiency of the $46 \times 26$ detector to the $38 \times 38$ detector for various sample sizes.

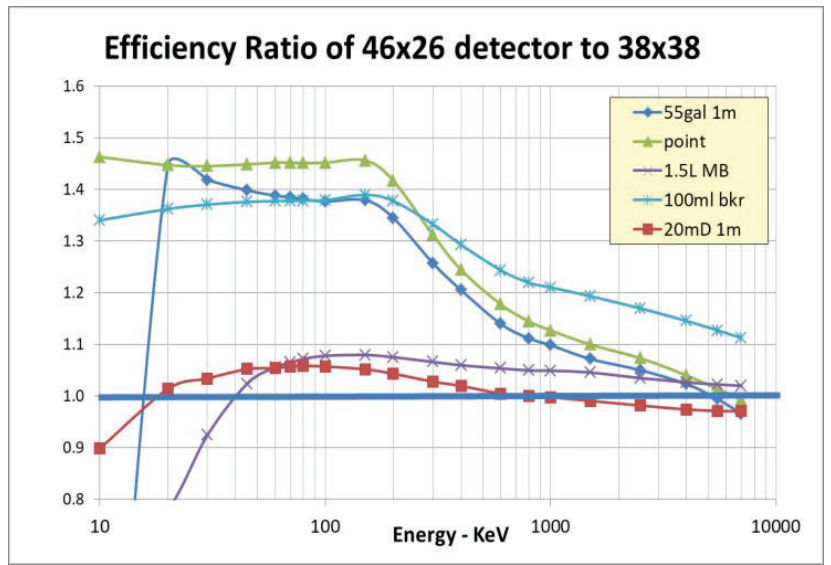

laboratory sample geometry. The $46 \times 26 \mathrm{~mm}$ detector has up to $25 \%$ higher efficiency.

The graphic on the right of Figure 4 explores this relationship for a wide range of other geometries. For all geometries where the source is predominately in front of the detector the efficiency for the high aspect-ratio $46 \times 26$ detector is always better up to very high energies. This true for both laboratory-type samples [ $100 \mathrm{ml}$ beaker, point-like sources] and common InSitu sources [200 liter drums]. Here, the $46 \%$ larger front surface area of the $46 \times 26$ detector more than makes up for the $32 \%$ shorter length. For other geometries where the source is both in front and at the sides [Marinelli Beakers, very large diameter sources] the $46 \times 26$ detector is still better at most all energies for most all gamma sources.

\section{Tests and Results}

We first explored the properties of Srl and CLYC in 2010-12 when executing a DHS contract on the development of a hand-held direction sensing spectrometer. The small crystals [25x25mm] available at that time had respectable resolution, typically $3.5-4 \%$ FWHM. But they all had high energy tailing, as shown in the top graphic of Figure 5. This is one of the better crystals from that project. While the low energy peaks were quite symmetric, the high-energy side of the Cs-137 peak is wider than the low energy side. Larger detectors and higher energies show even
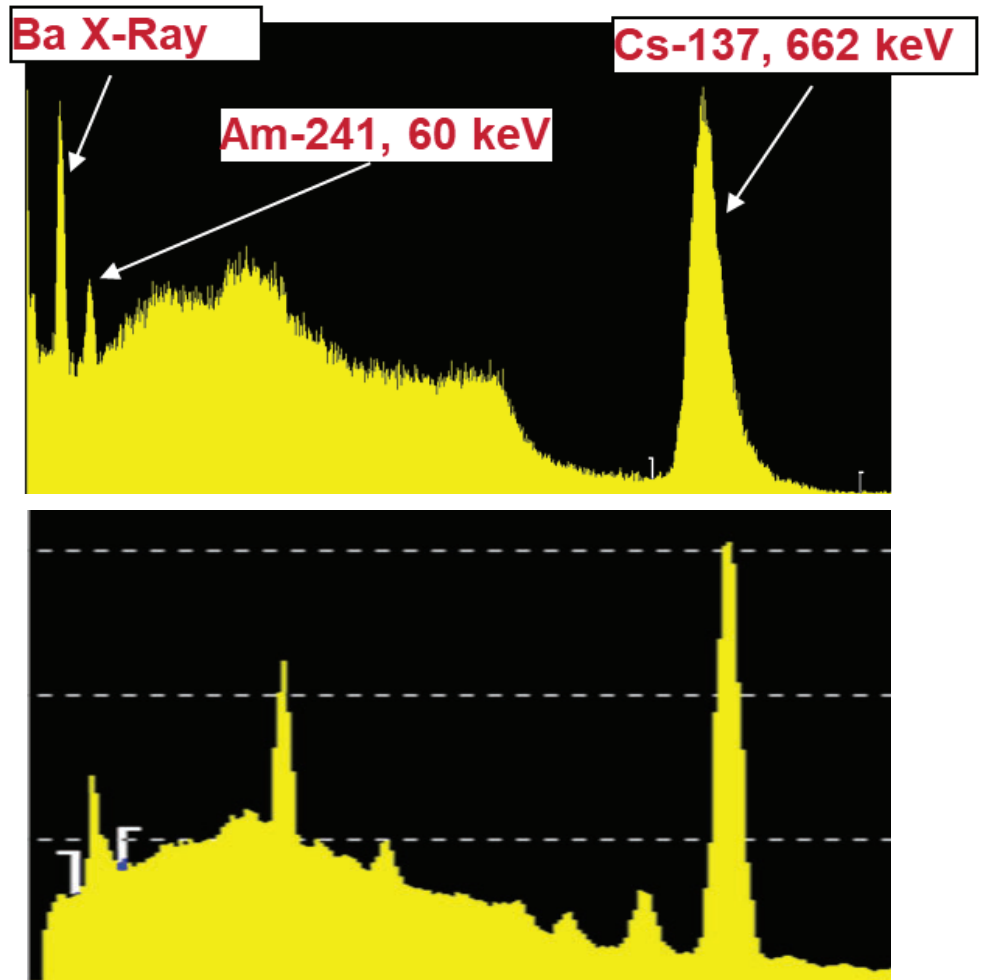

Figure 5 Top: 25x25mm Srl from 2011 DHS project. Bottom: detector from this study. Both have linear vertical counts scale and the same horizontal energy scale. more high-energy tailing. This makes it difficult for the peak fitting software to analyze the spectra, and 
can mask nearby higher energy peaks. Since older Ge detectors frequently had low-energy tailing due to charge loss, there are features in the peak fitting software to handle that; but no such features for highenergy tailing.

Fortunately today, this problem has mostly disappeared due to advances in material purity, better doping, better quality crystals, and better packaging. The bottom graphic in Figure 5 shows one of the detectors tested here - on the same energy scale. A thorium source is used in addition to Cs-137 which generates the peaks between $60 \mathrm{keV}$ and $662 \mathrm{keV}$. Notice that the Cs-137 peak is narrower [better resolution] and no observable high energy tailing. At high energies [e.g. $2614 \mathrm{keV}$ ] there still is some noticeable high energy tailing but fortunately there are fewer peaks up there, and the net peak area fitting process is still accurate.

The prime performance parameter is energy resolution - FWHM. Obtaining the best energy resolution requires setting the many parameters in the MCA signal processing chain at their optimum values. Due to the rather long decay time constant of the Srl scintillation light pulse, the MCA must integrate the signal long enough to capture most all this light - the more light captured, the higher the peak amplitude and the better the resolution. The Osprey Digital Signal Processing amplifier has two parameters that primarily affect this - Flat Top [FT] time and Rise Time [RT]. The FT time has a maximum limit of $3 \mathrm{uSec}$, and the RT has a maximum time of $5 \mathrm{uSec}$. It was quickly determined that these were not long enough for Srl. So a special version of the Osprey firmware was created that allows the Osprey RT to be increased to 50 uSec.

The test rig that was used is shown in Figure 6. A Cs-137 source is at the right with the radiation label. The detector is pointed to a case of Tungsten welding rods that have $2 \%$ Thorium. The Thorium and

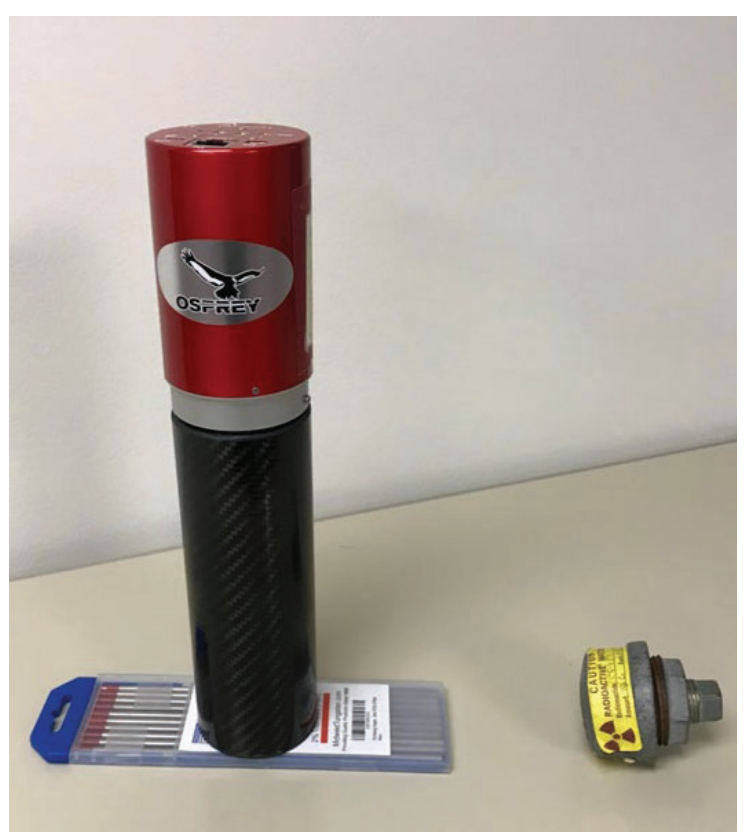

Figure 6 Test geometry used for these initial set of tests. Thoriated tungsten welding rods under detector and Cs137 source. daughters have many nice gammas all the way up to $2614 \mathrm{keV}$. And since the thorium is intimately mixed with the tungsten, the captured gammas cause the emission of the thorium X-ray which is at $60 \mathrm{keV}$ - the same energy as the important nuclide of Am-241. This test rig allows the examination of 7 energies that are all rather cleanly separated form near-by gammas. The Cs-137 source is there since that is commonly how performance of scintillators is referenced. The construction of the Cs-137 source is such that the 32 keV Ba X-rays do not escape.

For these tests the FT was set at the maximum of 3 uSec, and the RT was varied. At each new RT the gain was adjusted to keep the $\mathrm{keV} / \mathrm{channel}$ value constant.

The graph in Figure 7 shows how the FWHM in keV changes as the RT is changed. As the RT is increased the FWHM decreases. The improvement in FWHM is much more significant at high energies than at low energies. 


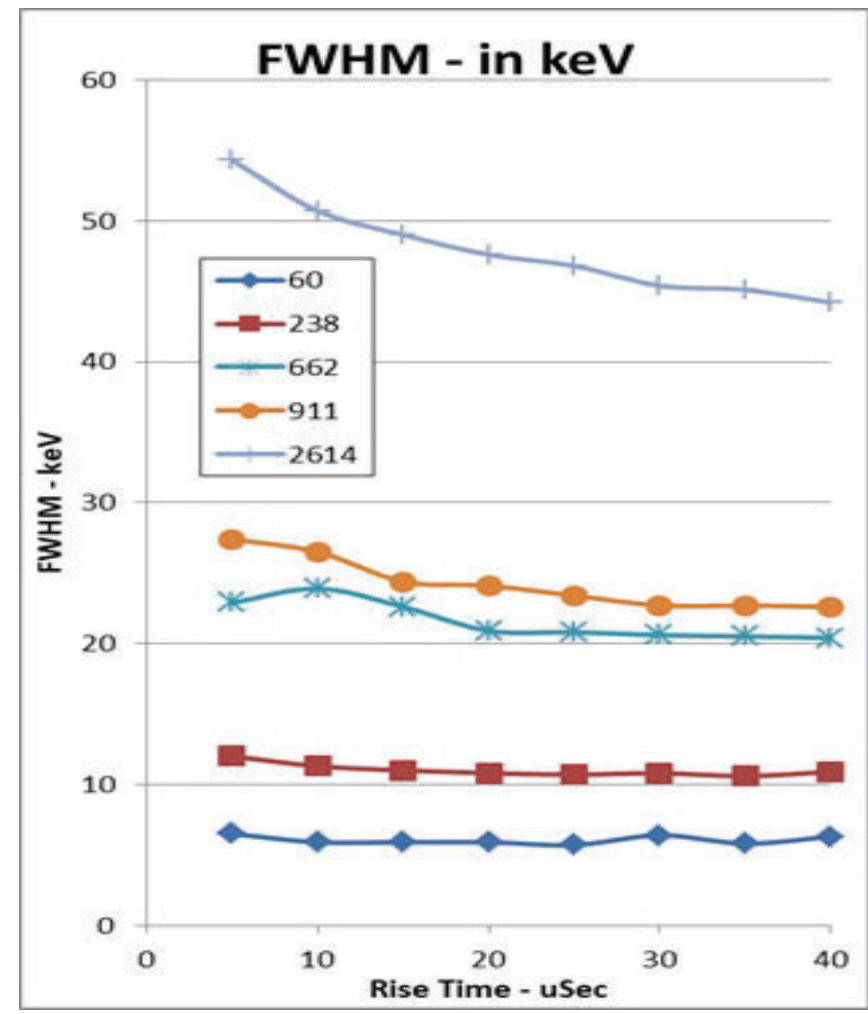

Figure 7 FWHM for 5 energies from 60 to $2614 \mathrm{keV}$ as the Rise Time is varied.

Another test that was performed to examine the impact of increasing count rates in the detector. With the detector still sitting on top of the Thorium welding rods, the Cs-137 source was moved stepwise closer to the detector. The total spectrum counts per second was recorded along with the $662 \mathrm{keV}$ Cs-137 peak centroid and \%FWHM. This is shown in Figure 9. The count rate was changed from around 100 to around 15,000 . The gain was quite stable, only changing a few channels [less than 3 $\mathrm{keV}]$ which is insignificant given the approximately $20 \mathrm{keV}$ FWHM. There was a slight increase of 2-3 keV in the FWHM value, not a very large value either. But the $\%$ deadtime did increase from $6 \%$ at $132 \mathrm{cps}$ to $41 \%$ at about $15,000 \mathrm{cps}$.

With modern digital signal processing MCAs like the Osprey, high \% dead times are not
One might be tempted to operate at the highest RT setting. However as the signal integrating time is increased, the number of signals that can be processed per second decreases. Figure 8 shows that even with no change in total count rate, the \% DeadTime is doubled when going from 5 uSec RT to 25 uSec RT.

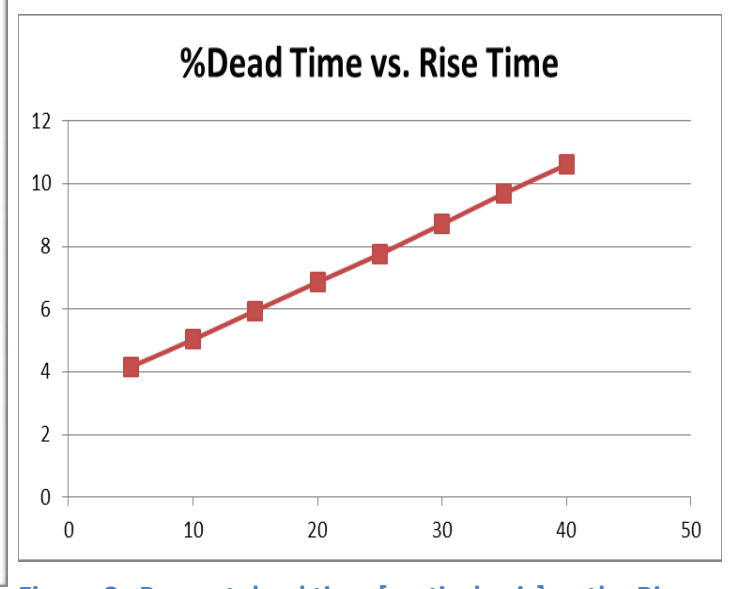

Figure 8 Percent dead time [vertical axis] as the Rise Time is changed [horizontal axis].

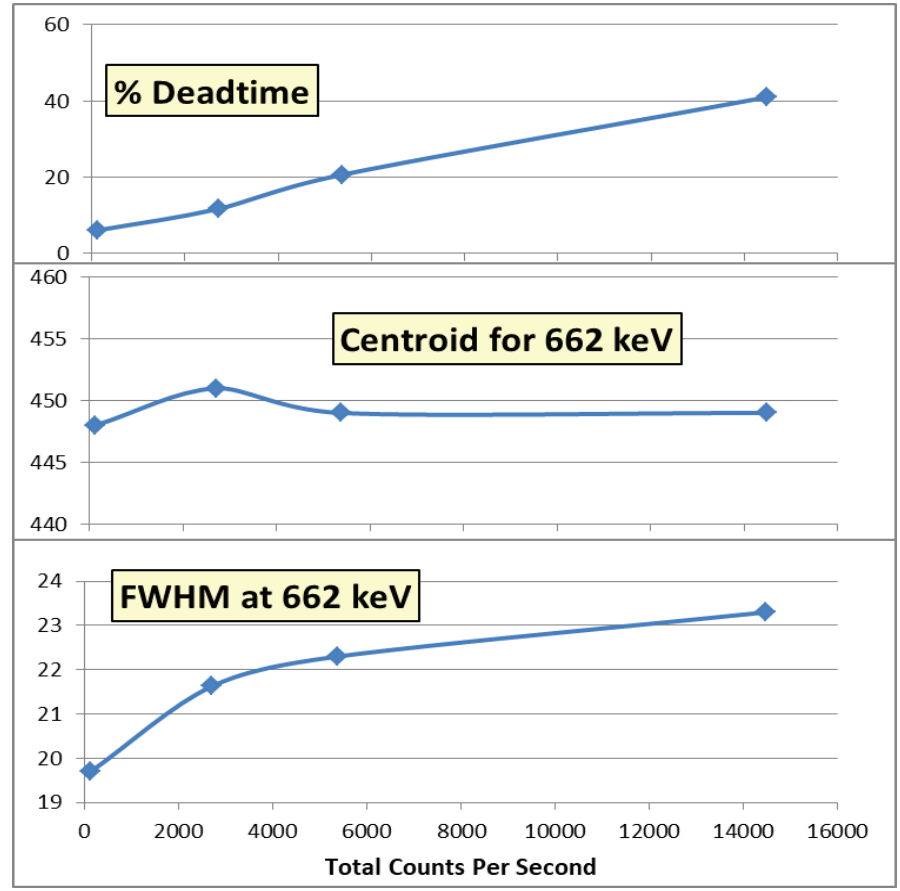

Figure 9 Change in \%DeadTime, peak centroid, and FWHM as the total CPS was increased. 
necessarily a problem, but it would seem prudent to carefully examine the accuracy of the live time before pushing Srl detectors much beyond the $20-25,000 \mathrm{cps}$ range.

This does point out one of the shortcomings of Srl - the long decay time. Other scintillators, notably $\mathrm{LaBr}$ have very short decay times, and are much better suited for high count rates, up to where limited by the performance of the PMT. For similar reasons, Srl scintillators are now as well suited for measurement situations where accurate signal timing is required.

\section{FWHM performance of Srl and other scintillators}

The following graphs in Figure 10 show the measured FWHM from these Srl tests and comparisons with the other scintillators. In comparison to $\mathrm{Nal}$, all of these enhanced resolution scintillators are better, with Srl being the best, just slightly better than LaBr. The graphic on the left is the high energy range up to $1400 \mathrm{keV}$, while the graphic on the right is an expanded view of the same data in the $50-300 \mathrm{keV}$ range.
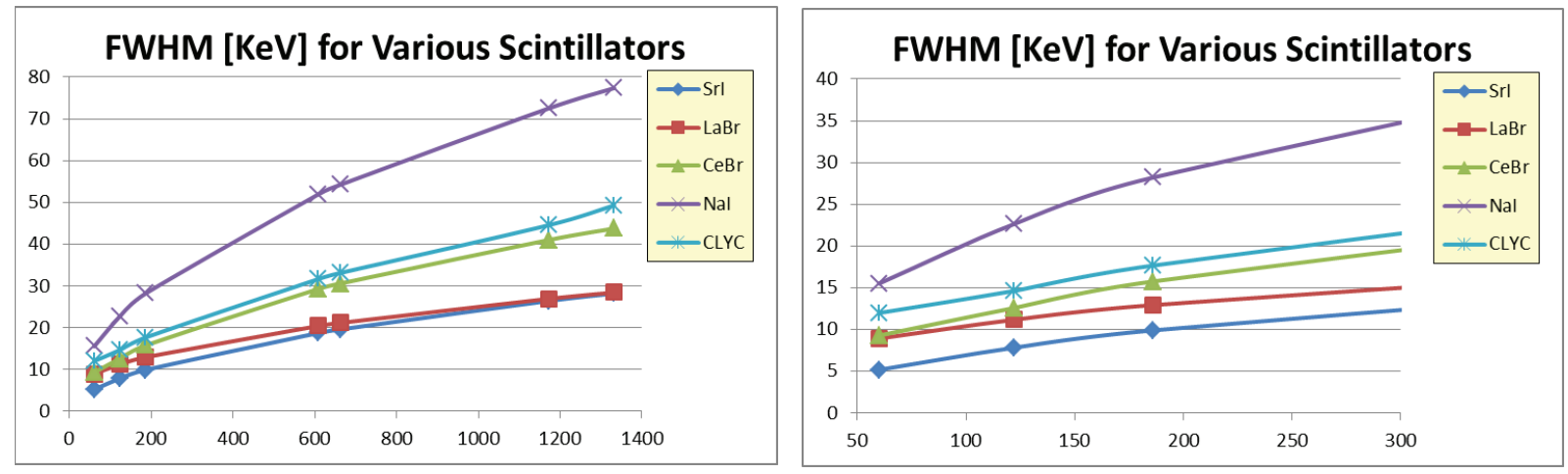

Figure 10 FWHM in keV for various scintillators as a function of gamma energy. The graph on the right is a expanded view of the graphs on the left.

The low energy range up to $\sim 300 \mathrm{keV}$ has some important nuclides that are commonly required to be measured. These include Am241, U-235, U-238, Pu-239, and most all nuclear medicine radionuclides. The expanded range chart on the right of Figure 10 clearly shows the advantage of Srl to separated peaks in this crowded low energy area. This performance can be even improved with better PMTs and better matching of signal processing electronics. The graphic in Figure 11 shows the same detectors with these improved features, as measured by the Srl supplier, CapeSym. The upper line is the same data as in Figure 10.

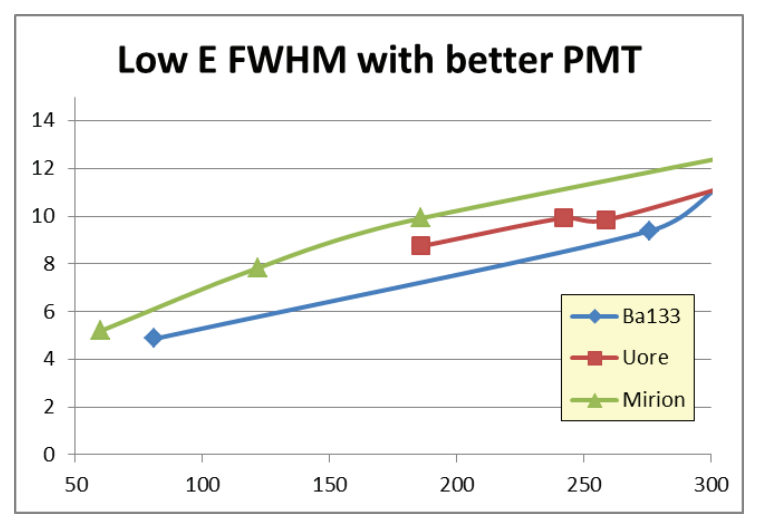

Figure 11 FWHM for low energy nuclides when measured with higher performance PMTs 
While there is a small dependence on light output from all scintillators as a function of temperature that variation is overwhelmed by the temperature dependence of the PMT. Figure 12 shows data from CapeSym on the variation of centroid position [gain] and FWHM as the detector and similar PMT are going through a temperature cycle test.

The detector and PMT were in an environmental chamber. The temperature was changed from -25 $\operatorname{deg} \mathrm{C}$ to $+55 \operatorname{deg} \mathrm{C}$. The right-hand scale shows the FWHM change with the values expressed as Percent at $662 \mathrm{keV}$. While the FWHM does increase slightly as the temperature is increased, it only changes very little around the nominal room temperature value of $3.2 \%$ [21 keV]. These changes are well within the ability of the Genie gamma spectral

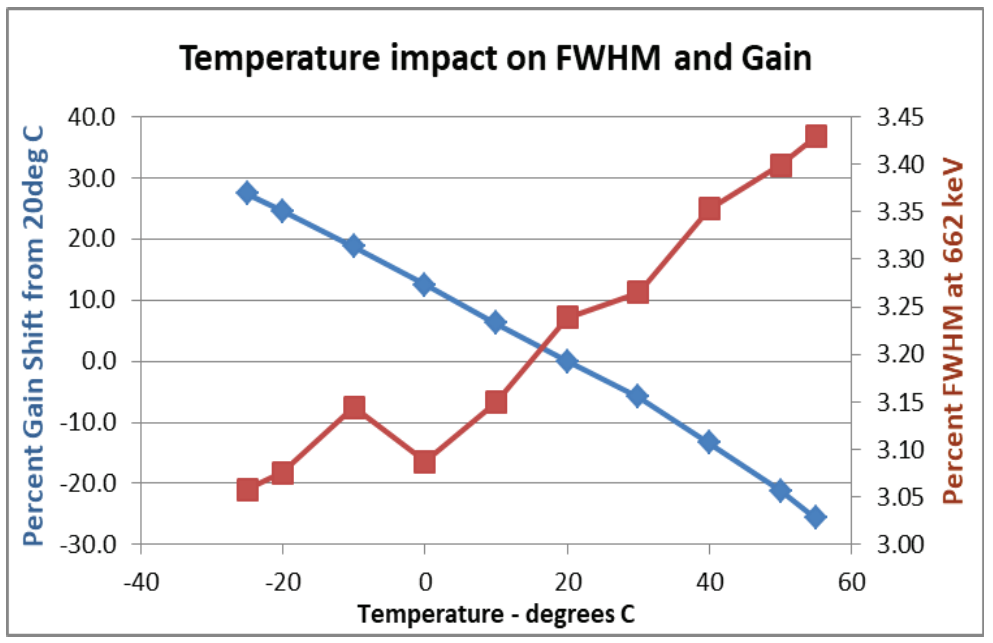

Figure 12 Temperature sensitivity of FWHM and Gain. analysis software to handle.

However the gain changes very significantly going from $25 \%$ higher at -25 degC to $30 \%$ lower at +55 degC. The typical parameters in the Genie gamma spectral analysis software will typically correctly assign the nuclide identity to a peak as long as it is within 1 FWHM of the expected position. For these enhanced resolution scintillators the reduced FWHM requires more gain stability than the common $\mathrm{Nal}$ detector. The "within $1 \mathrm{FWHM"} \mathrm{requirement} \mathrm{means} \mathrm{for} \mathrm{proper} \mathrm{nuclide} \mathrm{identification} \mathrm{performance,} \mathrm{the}$ temperature must be kept within $+/-4.5 \mathrm{degC}$ [ $8 \mathrm{deg} F]$ of the energy calibration temperature.

Alternately, and preferably, an automatic gain stabilization method should be implemented, such as the Canberra LED stabilization process that we have already implemented on $\mathrm{Nal}, \mathrm{LaBr}$, and $\mathrm{CeBr}$ scintillators.

\section{Minimum Detectable Activity estimations.}

This last section attempts to predict the performance of Srl to measure low activity samples and objects. Since there are an infinitely wide variety of sample detector geometries to choose from and since Nal is the most common scintillator, it was decided to simplify this section by computing these MDAs relative to the MDA of Nal.

Factors affecting MDA are:

- Detection Efficiency: the higher the detection efficiency the lower the MDA. The MDA is inversely proportional the detection efficiency.

- FWHM: the lower the FWHM the lower the background under the peak and the lower the MDA. The MDA is proportional to the square root of the FWHM

- Background: the lower the background under the peak and the lower the MDA. For equal size detectors [without any internal contamination], the background is approximately proportional to the mass of the detector. The MDA is proportional to the square root of the mass of the detector. 
Combining the relationships above, the MDA is proportional to:

$$
\sqrt{(\text { detector mass }) x(F W H M)} / \text { peak efficiency }
$$

Using this relationship a value for each of the enhanced resolution detectors was calculated and ratio to the computed value for Nal was determined.

For this exercise we

"assumed" that somehow the natural radioactivity of LaBr could be eliminated.

Table 2 shows the numerical data, and the Figure 13 graphic shows it visually.

For all energies these enhanced resolution scintillators have lower MDAs than Nal, with the exception of CLYC at low energies.

For all energies, Srl has the lowest MDA. Srl is slightly better than the [non-existant nonradioactive] $\mathrm{LaBr}$ at medium to high energies and significantly better than all of the scintillators at the important low-medium energy range.

\section{Conclusion}

Srl has been tested and shown to have the best energy resolution of the group of enhanced-resolution detectors.

This energy resolution combined with the low internal radioactivity background make it best currently available enhanced resolution detector for low MDAs and optimum nuclide identification.

A high-aspect ratio size detector shape has been shown to have up to $25 \%$ higher efficiency and therefore $25 \%$ lower MDA than a conventional shape where the diameter is equal to the height of the detector.

Limitations have been found in the current parameters available for adjustment in the Osprey digital MCA and software fixes have been prototyped and tested. 
Limitations have also been identified in the temperature response from the scintillator-PMT combination. This probably not an issue for laboratory sample counting applications, but would require some means to limit temperature fluctuations or to stabilized the gain for field applications.

This combination of computations and test results has shown that Srl scintillation detectors will have the lowest MDA of these enhanced resolution detectors, if all detectors are the same size.

Srl scintillators should be especially useful in low energy measurements of low activity samples. 